\title{
HIV prevalence among HIV-exposed infants participating in a programme for the prevention of mother-to-child transmission at Albazine Health Centre in Maputo
}

\author{
A Moiane, BSc Hons; A E Chongo, MSc; M N Cuco, BSc Hons; C Faiela, MPH \\ Department of Biological Sciences, Faculty of Science, Eduardo Mondlane University, Maputo, Mozambique
}

Corresponding author: A E Chongo (alda.chongo@uem.ac.mz)

\begin{abstract}
Background. In 2002, Mozambique introduced an inclusive and complete programme for the prevention of mother-to-child transmission (PMTCT) of HIV, comprising several components of continuous healthcare.

Objectives. To describe HIV infection rates in HIV-exposed children participating in a PMTCT programme.

Methods. The study comprised the retrospective collection of data of 10097 pregnant women and 2269 HIV-exposed infants who attended the Albazine Health Centre in Maputo, Mozambique, between 2012 and 2015. Descriptive statistics were used to present HIV prevalence and compliance with antiretroviral therapy (ART) in pregnant women, as well as HIV prevalence in infants at the ages of 1 month and between 9 and 18 months.

Results. HIV testing coverage in pregnant women ranged from $84 \%$ in 2012 to $97 \%$ in 2015 . HIV prevalence in pregnant women decreased from $16.7 \%$ in 2012 to $9.1 \%$ in 2015. Polymerase chain reaction (PCR) testing coverage in 1-month-old infants ranged from $62 \%$ in 2013 to $76 \%$ in 2015. Positive PCR results ranged from 4.4\% in 2012 to $1.5 \%$ in 2015. The HIV prevalence in children between 9 and 18 months of age reached $6.8 \%$ in 2015 . The HIV infection rate was lower (5.6\%) among children who were exclusively breastfed than in those who received formula feeds $(20.6 \%)$ or mixed feeding $(29 \%)$.

Conclusion. Despite improvements in ART coverage, HIV prevalence in infants remains a cause of concern. Further studies should include following up mother-infant pairs to obtain a more accurate picture of ART adherence and infant feeding outcomes.
\end{abstract}

S Afr J Child Health 2020;14(4):171-173. https://doi.org/10.7196/SAJCH.2020.v14i4.1694

In Mozambique, the prevalence of HIV infection in children under 5 years of age was $\sim 2.5 \%$ in 2016 and contributed $10 \%$ to infant mortality. ${ }^{[1]}$ It is the third largest cause of morbidity after malaria and neonatal deaths. ${ }^{[1]}$

In children, mother-to-child transmission (MTCT) remains the most common form of HIV transmission, with $95 \%$ of infected children acquiring the virus through vertical transmission; the remaining $5 \%$ are infected through contaminated blood transfusions, rape and early sexual activity. ${ }^{[1]}$

Therefore, to control MTCT, it was important to introduce an inclusive and complete programme for the prevention of motherto-child transmission (PMTCT), comprising several components of continuous healthcare, in Mozambique. ${ }^{[1]}$ Initially (2002), only eight health centres offered antiretroviral therapy (ART) for pregnant women. However, the number of sites increased until the PMTCT programme acquired national status in 2004, and in 2006 a plan for accelerated expansion was introduced. ${ }^{[1]}$ Given the economic situation, cultural context and other relevant factors, the health systems of countries implementing PMTCT programmes can select one of three options ( $\mathrm{A}, \mathrm{B}$ or $\mathrm{B}+$ ) according to their respective advantages and disadvantages. ${ }^{[2,3]}$ In 2013, Mozambique introduced Option $\mathrm{B}+$, which simplified the provision of ART, standardised therapeutic regimes and ensured the availability of more effective prophylaxis for the PMTCT programme. ${ }^{[1]}$ With Option $\mathrm{B}+$, pregnant women start $\mathrm{ART}$ as soon as they are diagnosed as HIV-positive, regardless of gestational stage and CD4 count, and remain on lifelong ART. ${ }^{[2,3]}$ With this option, infants receive nevirapine daily or azidothymidine twice a day from birth to age 4 - 6 weeks, regardless of feeding method. ${ }^{[2]}$ Exclusive breastfeeding is recommended for the first 6 months of the infant's life irrespective of HIV status, after which complementary foods or drinks can be introduced. Breastfeeding should, however, continue till the age of 2 years or beyond. ${ }^{[4]}$

The $\mathrm{B}+$ option offers several advantages, including greater efficiency and greater agreement with the Treatment 2.0 Framework for Action, ${ }^{[2]}$ simplified and optimised use of ART and a standardised first-line treatment regimen. Moreover, it eliminates the necessity for multiple orientations and training. ${ }^{[2]}$ However, disadvantages of the $\mathrm{B}+$ option are that it needs to be evaluated in both programme and field scenarios, with an assessment of community acceptability and human rights protection. ${ }^{[2,3]}$

In 2011, Mozambique adopted the Global Plan for Elimination of MTCT of HIV and set its target to reduce MTCT to less than $5 \%$ by the year $2015 ; ;^{[1]}$ the target date was subsequently extended to 2017. ${ }^{[1]}$ The programme also envisaged $90 \%$ ART coverage to HIV-seropositive pregnant women. ${ }^{[1]}$ Monitoring ART coverage and HIV infection rates in HIV-exposed children can identify trends and assist in providing valuable information that would enable programme managers to prioritise actions. The aim of the present study was to describe HIV infection rates in HIV-exposed infants 
participating in a PMTCT programme at the Albazine Health Centre (AHC) in Maputo, Mozambique.

\section{Methods \\ Study setting}

This descriptive study was conducted at the AHC in Maputo, Mozambique, which is a level 2 health unit located in the Albazine neighbourhood. It provides healthcare services to the neighbourhoods of Albazine, Magoanine C, Mutanhane and Guava.

\section{Data collection}

A retrospective analysis of health records was performed from a total of 10097 pregnant women and 2269 HIV-exposed infants for the period 1 January 2012 - 31 December 2015. The study was approved by the institutional ethics committee (Comité Institucional de Bioética em Saúde da Faculdade de Medicina \& Hospital Central de Maputo), which waived the requirement for informed consent owing to the retrospective nature of the study. Only complete data sets of pregnant women were included in the study. The following indicators were collected: (i) HIV testing coverage in pregnant women; (ii) HIV prevalence in pregnant women; (iii) ART coverage in pregnant women; (iv) HIV testing coverage in children in the first month after birth and between 9 and 18 months of age; $(v)$ HIV prevalence in children in the first month after birth and between 9 and 18 months of age; and ( $v i)$ infant feeding options and HIV prevalence.

On-site laboratory records, antenatal records and post-natal follow-up of HIV-exposed children were collected and evaluated. Secondary data used in this study were obtained from laboratory analysis of blood samples from pregnant women and infants born to HIV-infected women (HIV-exposed infants). In the case of 1-month-old children, blood was collected at the AHC and sent to the National Molecular Virology Laboratory of the National Institute of Health, as well as to the Molecular Virology Laboratory of the General Hospital of Mavalane for HIV diagnosis using polymerase chain reaction (PCR). For children aged 9 - 18 months and pregnant women, blood was collected and tested at the AHC using rapid tests (Alere Determine (Orgenics Ltd., Israel) and Uni-Gold Recombigen (Trinity Biotech PLC, Ireland)).

In relation to infant feeding options, an infant was classified as being exclusively breastfed, mixed-fed or formula-fed according to the mothers' responses as registered at the time of the PCR test. To describe the infant feeding option in relation to HIV infection, we included only serology data as obtained at 9 months of age. Pregnant women were tested for HIV infection on the day of opening their prenatal record; testing was not performed for those who claimed to be HIV-seropositive.

Descriptive statistics were used to explore HIV prevalence and ART compliance in pregnant women, as well as HIV prevalence in infants at the ages of 1 month and $9-18$ months.

\section{Results}

HIV testing coverage in pregnant women ranged from $79.8 \%$ (2013) to 97\% (2015) (Table 1). HIV prevalence decreased from $16.7 \%$ in 2012 to $9.1 \%$ in 2015 . The ART coverage improved from $45.8 \%$ in 2012 to $100 \%$ in 2013 and the following years. PCR coverage in 1-month-old infants ranged from $62.4 \%$ to $76.7 \%$, with the lowest coverage in 2013. Positive PCR ranged from $4.4 \%$ in 2012 to $1.5 \%$ in 2015 (Table 2).

Despite good coverage and $90 \%$ serology in most years, HIV prevalence among children aged 9 - 18 months still ranged between
Table 1. Prevalence of HIV infection and uptake of antiretroviral therapy among pregnant women presenting to the Albazine Health Centre, Mozambique, from 2012 to 2015 Year Women Women Seropositive ART initiated, consulted, $n$ tested, $n(\%)$ tests, $n(\%) \quad n(\%)$

\begin{tabular}{lllll}
\hline 2012 & 2767 & $2341(84.6)$ & $391(16.7)$ & $179(45.8)$
\end{tabular}

$\begin{array}{lllll}2013 & 2160 & 1724(79.8) & 222(12.9) & 222(100)\end{array}$

$20142580 \quad 2364(91.6) \quad 362(15.3) \quad 362(100)$

$20152590 \quad 2511(97) \quad 229(9.1) \quad 229(100)$

Total $10097 \quad 8940(88.5) \quad 1204(13.5) \quad 992(82.4)$

$\mathrm{ART}=$ antiretroviral therapy.

Table 2. HIV infection screening in HIV-exposed infants at the Albazine Health Centre, 2012 - 2015

\begin{tabular}{llllll}
\hline Year & $\begin{array}{l}\text { HIV- } \\
\text { exposed } \\
\text { infants, } \\
n\end{array}$ & $\begin{array}{l}\text { PCR } \\
\text { testing, }\end{array}$ & $\begin{array}{l}\text { Positive } \\
\text { PCR } \\
\text { result, } \\
n(\%)\end{array}$ & $\begin{array}{l}\text { Serology } \\
\text { testing, } \\
n(\%)^{*}\end{array}$ & $\begin{array}{l}\text { Positive } \\
\text { serology } \\
\text { result, } \\
n(\%)\end{array}$ \\
\hline 2012 & 646 & $451(69.8)$ & $20(4.4)$ & $630(97.5)$ & $33(5.2)$ \\
2013 & 486 & $303(62.4)$ & $19(6.3)$ & $472(97.1)$ & $28(5.9)$ \\
2014 & 531 & $372(70.1)$ & $9(2.4)$ & $521(98.1)$ & $47(9.0)$ \\
2015 & 606 & $465(76.7)$ & $7(1.5)$ & $463(76.4)$ & $31(6.8)$ \\
Total & 2269 & $1591(70.1)$ & $55(3.5)$ & $2086(91.9)$ & $139(6.7)$ \\
PCR = polymerase chain reaction \\
*PCR testing was performed in 1-month-old infants. Serology testing was \\
performed at the age of 9- 18 months.
\end{tabular}

$5 \%$ and $10 \%$. The highest prevalence in this group (9\%) was seen in 2014 , followed by a decrease to $6.8 \%$ in 2015 .

Infant feeding records $(n=923)$ showed that the majority of mothers opted for exclusive breastfeeding rather than formula or mixed feeding (Fig. 1). The HIV infection rate was $5.6 \%$ for children who were exclusively breastfed, followed by a prevalence of $20.6 \%$ and $29 \%$ among formula-fed infants and those who received mixed feeding, respectively.

\section{Discussion}

Results showed that ART coverage improved from 45.8\% in 2012 to $100 \%$ since 2013 . This improvement may be linked to the $\mathrm{B}+$ option of the PMTCT programme that was implemented in Mozambique in 2013. With this option, all HIV-positive pregnant women were eligible for ART once diagnosed. The reduction in viral load associated with ART consequently decreased the risk of vertical transmission.

HIV prevalence among infants of 1 month of age fell from $4.4 \%$ to $1.5 \%$ between 2012 and 2015. The B+ option and improved coverage may have contributed to the observed reduction in HIV infection rates post delivery. HIV prevalence among 9 -18-month-old children was $6.8 \%$ in 2015 , which was still above the national target of $5 \%$. To achieve the national and international goal for reducing MTCT, the AHC should explore how they can encourage women more effectively to adhere to the PMCT programme and consider performing PCR testing in infants in the first month of life, followed up with serology testing at $9-18$ months. Monitoring ART adherence and the associated viral load during pregnancy and post partum may further improve the programme's effectiveness, especially to ensure suppression before delivery and during breastfeeding. 


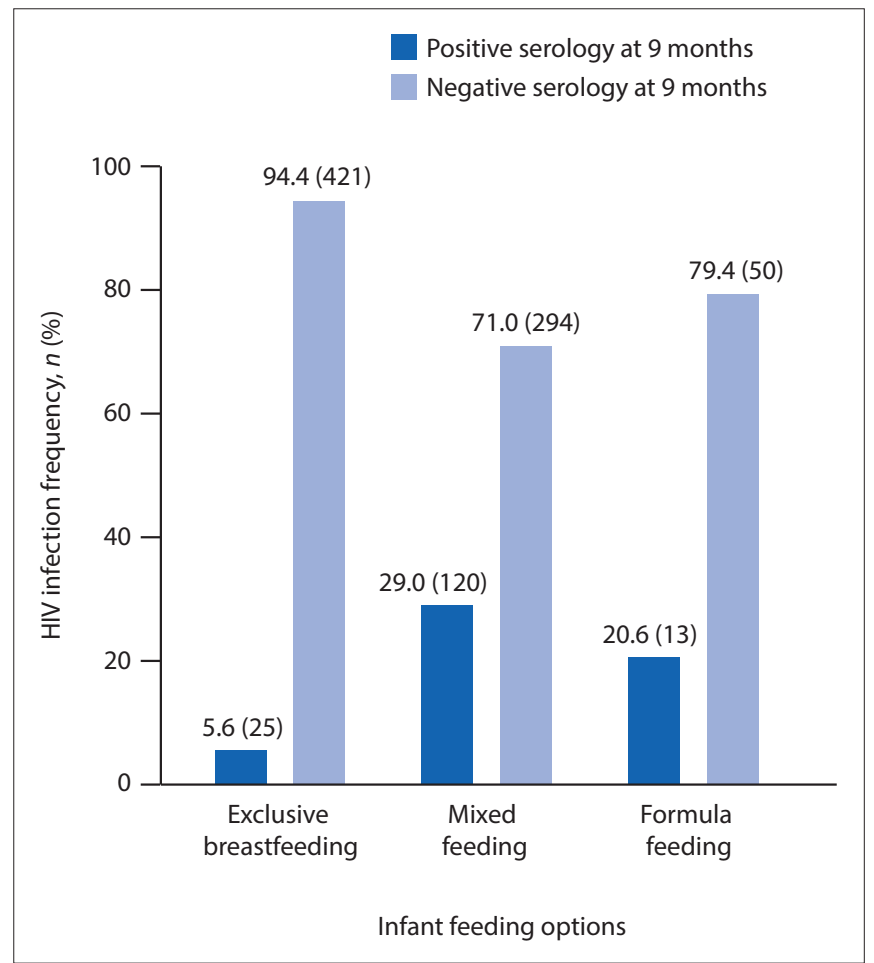

Fig. 1. HIV infection frequency according to infant feeding options in children at 9 months of age $(\mathrm{n}=923)$.

The HIV infection rate was lower among children who were exclusively breastfed, followed by those who received formula milk or mixed feeding. As we had no data concerning drug adherence in the context of $100 \%$ ART coverage nor on the number of women who achieved viral suppression, we were unable to test any possible association between infant feeding options and HIV infection rate. However, other studies ${ }^{[5,6]}$ have shown that exclusive breastfeeding is associated with a lower frequency of HIV than in a mixed-feeding model.
As data from this study were not collected as mother-infant pairs, except for the infant feeding options, we were not able to correlate prevalence rates in infants with ART coverage.

\section{Conclusion}

Despite improvements in ART coverage, HIV prevalence in infants remains a cause of concern. Further studies should include following up infant-mother pairs for a more accurate understanding of ART adherence and infant feeding outcomes.

Declarations. This manuscript was submitted in partial fulfilment of the requirements for a Masters degree at the Eduardo Mondlane University. Acknowledgements. We would like to thank the staff of the Albazine Health Centre for providing the data for analysis.

Author contributions. All authors contributed equally to the research and manuscript development.

Funding. None.

Conflicts of interest. None.

1. Ministério da Saúde. Programma Nacional de Controlo de ITS, HIV/SAID. Relatório Anual das Actividades Relacionadas ao HIV/SIDA 2019. http://www. misau.gov.mz/index.php/relatorios-anuais (accessed 26 February 2020).

2. World Health Organization. The treatment 2.0 framework for action: Catalysing the next phase of treatment, care and support. Geneva: WHO, 2011. https:// www.unaids.org/sites/default/files/media_asset/20110824_JC2208_outlook_ treatment2.0_en_3.pdf

3. Liu L, Johnson HL, Cousens S, et al. Global, regional, and national causes of child mortality: An updated systematic analysis for 2010 with time trends since 2000. Lancet 2012;379(9832):2151-2161. https://doi.org/10.1016/s01406736(12)60560-1

4. Decker S, Rempis E, Schnack A, et al. Prevention of mother-to-child transmission of HIV: Postpartum adherence to Option B+ until 18 months in Western Uganda. PLoS One 2017;12(6):e0179448. https://doi.org/10.1371/journal pone.0179448

5. Children \& Aids. Mozambique Tratamento Antiretroviral. https://www. childrenandaids.org/Mozambique_Tratamento-Antiretroviral_2016 (accessed 25 November 2019).

6. Anoje C, Aiyenigba B, Suzuki C, et al. Reducing mother-to-child transmission of HIV: findings from an early infant diagnosis program in south-south region of Nigeria. BMC Public Health 2012;12:184. https://doi.org/10.1186/1471-2458-12-184

Accepted 30 April 2020. 